\title{
Optimal Radiation of Body-Implanted Capsules
}

\author{
Denys Nikolayev, ${ }^{1, *}$ Wout Joseph, ${ }^{1}$ Maxim Zhadobov, ${ }^{2}$ Ronan Sauleau, ${ }^{2}$ and Luc Martens ${ }^{1}$ \\ ${ }^{1}$ Imec - Ghent University, Ghent 9052, Belgium \\ ${ }^{2}$ Univ Rennes, CNRS, Institut d'Électronique et de Télécommunications de Rennes, UMR-6164, F-35000 Rennes
}

(Dated: February 25, 2019)

\begin{abstract}
Autonomous implantable bioelectronics requires efficient radiating structures for data transfer and wireless powering. The radiation of body-implanted capsules is investigated to obtain the explicit radiation optima for $\mathbf{E}$ - and $\mathbf{B}$-coupled sources of arbitrary dimensions and properties. The analysis uses the conservation-of-energy formulation within dispersive homogeneous and stratified canonical body models. The results reveal that the fundamental bounds exceed by far the efficiencies currently obtained by conventional designs. Finally, a practical realization of the optimal source based on a dielectric-loaded cylindrical-patch structure is presented. The radiation efficiency of the structure closely approaches the theoretical bounds and shows a fivefold improvement over existing systems.
\end{abstract}

Miniature body-implanted devices offer powerful capabilities for medicine and clinical research enabling more precise diagnostics and treatment than ever before [1]. For instance, the emerging electroceuticals aim individual neural circuits that regulate the physiological processes to treat a wide range of illnesses [2]. Likewise, wireless powering makes the lifespan of implants practically unlimited; efficient theoretical approaches have been proposed recently [3-5]. Body-conformal surfaces can focus energy into tissues [6]. However, efficient radiating structures are required for body-implanted capsules to overcome existing limitations on powering, safety, and data transfer. These constraints prohibit us, for instance, to realize wireless neural interfaces $[7,8]$, implantable labon-a-chips [9], and surgical microbots [10].

Physical bounds on radiation efficiency $\eta$ of arbitrary sources in free space have been extensively studied [1115]. Effects of tissues on $\eta$ and optimal frequency $f_{\text {opt }}$ have been considered in [16] for body-implanted inductor sources and in [17] for infinitesimal magnetic dipole and current sources. It is still unclear whether magnetic B-coupled $\left(\mathrm{TE}_{10}\right)$ or electric E-coupled $\left(\mathrm{TM}_{10}\right)$ sources maximize $\eta$ in tissues. On the one hand, nearly all tissues are weakly diamagnetic. Therefore, negligible losses occur within the evanescent $\mathbf{B}$-field of $\mathrm{TE}_{10}$ (contrast this with $\mathrm{TM}_{10}$ where near-field energy is dissipated via dielectric relaxation [18]). On the other hand, high permittivity values of tissues act on $\mathbf{E}$-coupled sources, and higher $\eta$ can be achieved using dielectric loading [14].

In this Letter we obtain explicit radiation optima of body-implanted capsules equipped with arbitrary finitesized $\mathrm{TM}_{10}$ and $\mathrm{TE}_{10}$ sources. Optimal (in terms of $\eta[12])$ surface current density distributions on $\Sigma_{C}$ represent the sources (Fig. 1). Physical bounds on $\eta(f)$ strongly depend on the configuration and dielectric properties of the source region; this letter quantifies their effects for the first time. The results reveal that it is possible to outperform state-of-the-art designs by a factor of five in terms of radiation efficiency. Based on the results, we show that a dielectric-loaded cylindrical-patch source closely approaches this optimal efficiency.
Problem Formulation - A stratified sphere $\Omega_{P}$ of variable radius $R_{P}$ and of complex permittivity $\hat{\varepsilon}(r, \omega)=$ $\varepsilon^{\prime}-i \varepsilon^{\prime \prime}=\varepsilon_{0} \varepsilon_{r}(r, \omega)-i \sigma(r, \omega) / \omega$ (where $\varepsilon_{r}$ is the relative permittivity, $\sigma$ is the electrical conductivity, $\omega=2 \pi f$ is the angular frequency, and $f$ is the frequency) represents the tissues [Fig. 1(a)]. The thicknesses of 5-mm fat and 2-mm skin layers are kept constant throughout the study. The validity and limitations of this model are discussed in [19]. The EM field radiated from an arbitrary source inside $\Omega_{P}$ satisfies the inhomogeneous wave equation. In terms of the time-harmonic electric field $\mathbf{E}$ (time variations of the form $e^{i \omega t}$ ), it is expressed as $\nabla^{2} \mathbf{E}=i \omega \mu_{0} \mathbf{J}_{s}+i \omega \mu_{0} \sigma \mathbf{E}-\omega^{2} \mu_{0} \varepsilon_{0} \varepsilon_{r} \mathbf{E}$, where $\mathbf{J}_{s}$ is the source electric current density [20]. Taking into account the $z$-axial symmetry of the problem on Fig. 1, we reduce it to $\mathbf{R}^{2}$ assuming $\mathbf{E}(r, \varphi, z)=\overline{\mathbf{E}}(r, z) e^{-i m \varphi}$, where $m$ is the azimuthal mode number.

An arbitrary current density $\mathbf{J}$ (satisfying Maxwell's equations) flows through $\Omega_{P}$ and has values $\mathbf{J}_{s}$ on the cylindrical surface $\Sigma_{C}$ depicted on Fig. 1(b). The surface is defined by the variable length $L$ and radius $R_{C}$; the circumradius $a \equiv \sqrt{L^{2} / 4+R_{C}^{2}}$. To represent a generic (a)

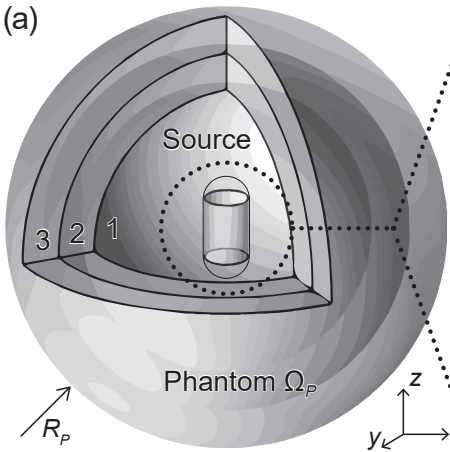

(b)

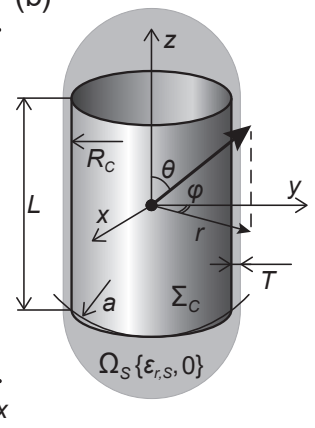

FIG. 1. Problem formulation (not to scale). (a) Stratified spherical model of body tissues (phantom) $\Omega_{P}$ around the source $\Omega_{S}$ : "1" is the muscle $\left\{\varepsilon_{r, 1}(\omega), \sigma_{1}(\omega)\right\}$, "2" is the 5mm layer of fat $\left\{\varepsilon_{r, 2}(\omega), \sigma_{2}(\omega)\right\}$, and " 3 " is the 2-mm layer of skin $\left\{\varepsilon_{r, 3}(\omega), \sigma_{3}(\omega)\right\}$. (b) Source region $\Omega_{S}$ defined as a current density distribution on the cylindrical surface $\Sigma_{C}$. 

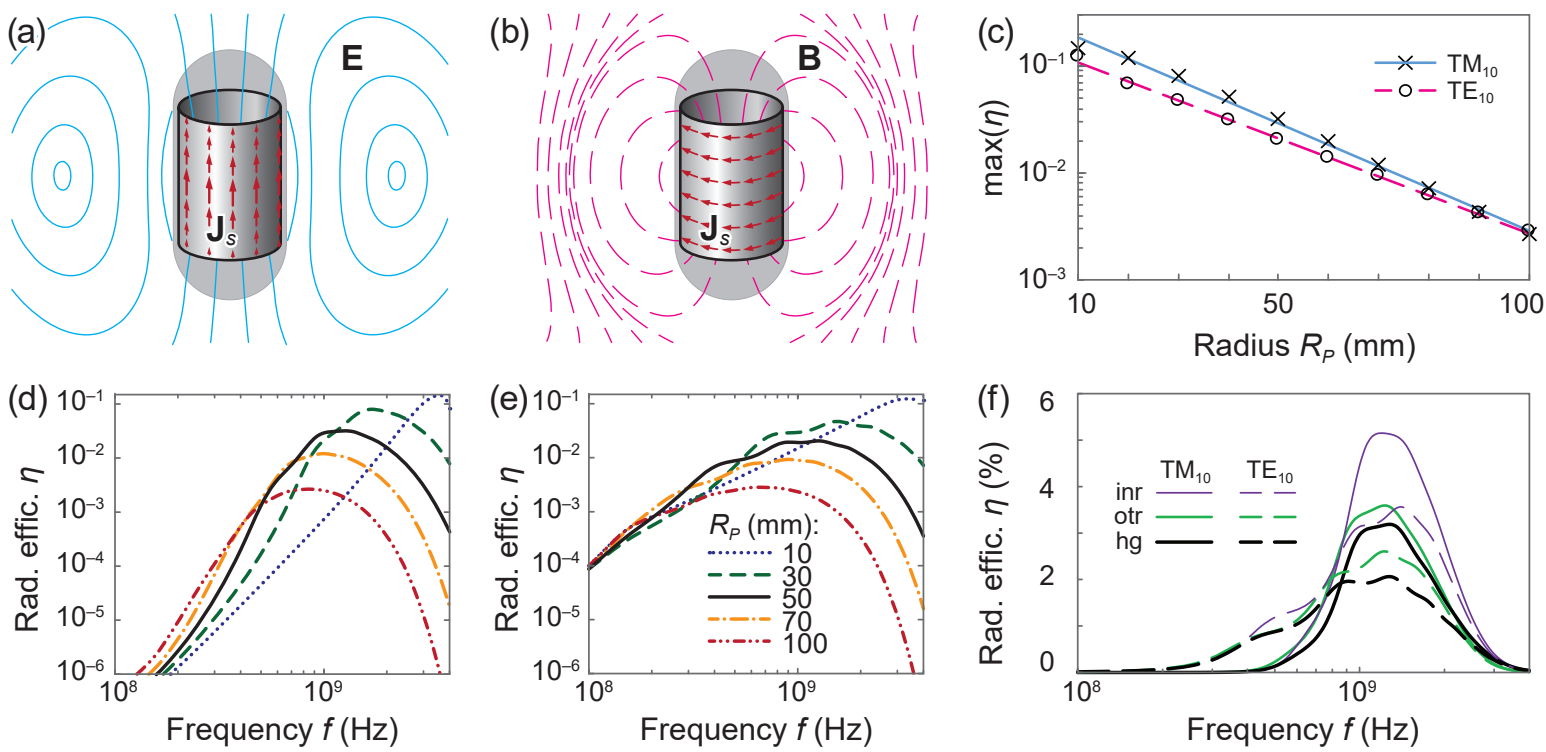

FIG. 2. (a) $\mathrm{TM}_{10}$ and (b) $\mathrm{TE}_{10}$ sources are defined as current density $\mathbf{J}_{s}$ distributions on the surface $\Sigma_{C}$; the contour lines depict the distribution of electric $\mathbf{E}$ and magnetic $\mathbf{B}$ fields, respectively (arb. units). (c)-(e) Implantation depth $\left(d \approx R_{P}-a\right)$ significantly affects the optimal frequency $f_{\text {opt }}$ and radiation efficiency $\eta$. (c) $\max (\eta)$ exponentially decays with implantation depth. (d)-(e) $\eta$ spectrum of (d) $\mathrm{TM}_{10}$ and (e) $\mathrm{TE}_{10}$. (f) Skin and fat layers [Fig. 1(a)] increase $\eta$ (compared to a homogeneous $R_{P}=50 \mathrm{~mm}$ phantom) for both sources by improving wave-impedance matching with free space. The effect is observed for both the layers placed inside (inr, $R_{P}=50 \mathrm{~mm}$ ) and outside (otr, $R_{P}=57 \mathrm{~mm}$ ) of the homogeneous (hg) phantom.

pill-shaped in-body device, a lossless (i.e. $\sigma=0$ ) region $\Omega_{S} \in \Omega_{P}$ encloses the surface $\Sigma_{C}$. The region consists of a cylinder of length $L$ and radius $R_{C}+T$ and two hemispheres of the same radius. Like so, the variable $T$ allows for evaluating the impact of shell (or superstrate) thickness on $\eta$. The permittivity $\varepsilon_{r, S}$ of $\Omega_{S}$ accounts for the effect of dielectric loading $\left(\varepsilon_{r, S} \propto \eta\right.$ [19]). It is assumed that $\Omega_{S}$ is composed of non-magnetic materials.

The surface current density $\mathbf{J}_{s}(r, \varphi, z)$ is defined on $\Sigma_{C}$ for $\mathrm{TM}_{10}$ [Fig. 2(a)] and TE 10 [Fig. 2(b)] modes as

$$
\begin{aligned}
\mathbf{J}_{s, \mathrm{TM}_{10}} & =[0,0, \cos (\pi z / L)], \\
\mathbf{J}_{s, \mathrm{TE}_{10}} & =(0,1,0) .
\end{aligned}
$$

The radiation efficiency $\eta$ is derived from the conservation of energy [20]. Poynting's theorem states that $P_{s}=P_{e}+P_{d}+i 2 \omega\left(\bar{W}_{m}-\bar{W}_{e}\right)$, where $P_{s}=$ $-0.5 \iiint_{\Omega_{S}}\left(\mathbf{H}^{*} \cdot \mathbf{M}_{i}+\mathbf{E} \cdot \mathbf{J}_{i}^{*}\right) d v$ is the supplied power, $P_{e}=\oiiint_{\Sigma_{R}}\left(0.5 \mathbf{E} \times \mathbf{H}^{*}\right) \cdot d \mathbf{s}$ is the exiting power, and $P_{d}=0.5 \iiint_{\Omega_{P}} \sigma|\mathbf{E}|^{2} d v$ is the dissipated power. In this way, we calculate the radiation efficiency as

$$
\eta \equiv \Re\left(P_{e}\right) / \Re\left(P_{s}\right) .
$$

The peak of $\eta(f)$ defines the optimal frequency $f_{\text {opt }}$ as $\eta\left(f_{\text {opt }}\right) \equiv \max (\eta)$.

Note that for a real-life antenna, the total radiation efficiency also includes a mismatch loss as $\eta_{\text {tot }}=$ $\eta\left(1-\left|\Gamma_{A}\right|^{2}\right)$, where $\Gamma_{A}$ is the reflection coefficient at the antenna feed. In this letter, we consider $\Gamma_{A}=0$.
Numerical Results - Studying $\eta(f)$ of the formulated problem requires solving the second-order linear partial differential equation. We use the fully-adaptive $h p$-finite element method implemented with the in-house code Agros2D [21]. The $h p$-adaptivity algorithm is set to maintain the total-energy relative error below $10^{-4}$. Additional verification ensures the power-balance residual $\max (\delta P) \equiv \max \left[\left(P_{s}-P_{e}-P_{d}\right) / P_{s}\right] \leq 10^{-3} \forall f$.

To represent the tissue dispersion, we use the fourregion Cole-Cole model $\hat{\varepsilon}(\omega)=\varepsilon^{\prime}-i \varepsilon^{\prime \prime}=\varepsilon_{\infty}+$ $\sum_{n=1}^{4}\left(\varepsilon_{s}-\varepsilon_{\infty}\right)_{n} /\left[1+\left(i \omega \tau_{n}\right)^{\left(1-\alpha_{n}\right)}\right]+\sigma_{i} /\left(i \omega \varepsilon_{0}\right)$. The parameters of the model for different tissues have been defined in [22] based on experimental data [23].

We start by calculating the effect on $f_{\text {opt }}$ and $\max (\eta)$ of the phantom radius $R_{P} \in[10,100] \mathrm{mm}$ that is asymptotic to the source implantation depth $d \approx R_{P}-a$. The source geometry is $L=10 \mathrm{~mm}, R_{C}=4 \mathrm{~mm}$, and $T=0.5 \mathrm{~mm}$ (average properties of existing capsules [1]), and $\varepsilon_{r, S}=\varepsilon_{r, 1}$ (Fig. 1). If not indicated otherwise, these source properties are used hereafter. Fig. 2(c) shows the maximum achievable efficiency as a function of $R_{P}$.

As can be seen in Figs. 2(d)-(e), the optimal frequency range follows a skew normal distribution within considered $f \in[0.1,4] \mathrm{GHz}$. For a given depth, $f_{\text {opt }}$ is the best compromise between the attenuation losses $\alpha \propto f$ on the one hand, and the wave-impedance mismatch at the tissue-air interface $|\Gamma| \propto f^{-1}$ as well as the source electrical size $k a$ (where $k$ is the wavenumber) on the other [17]. For $\mathrm{TM}_{10}$, the optimal frequency can be approxi- 

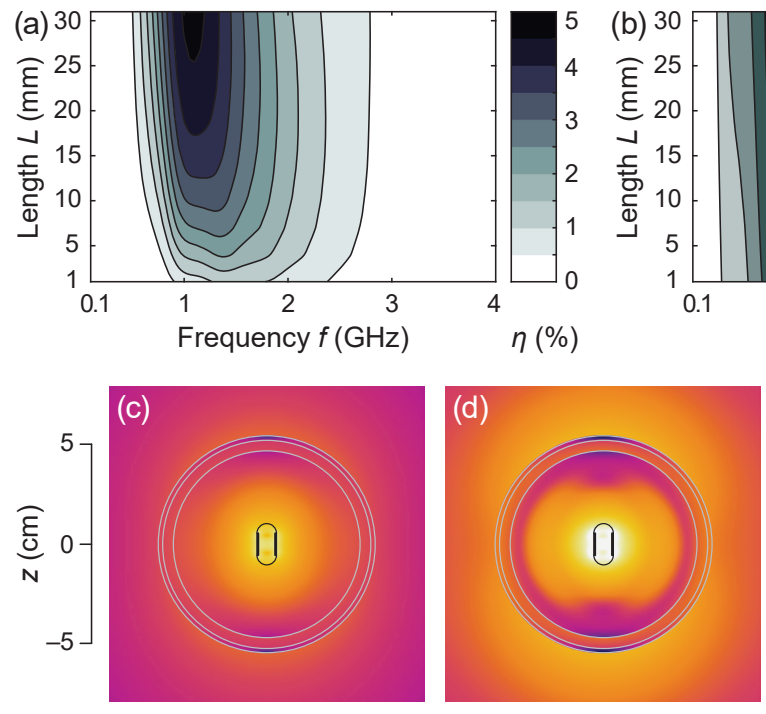
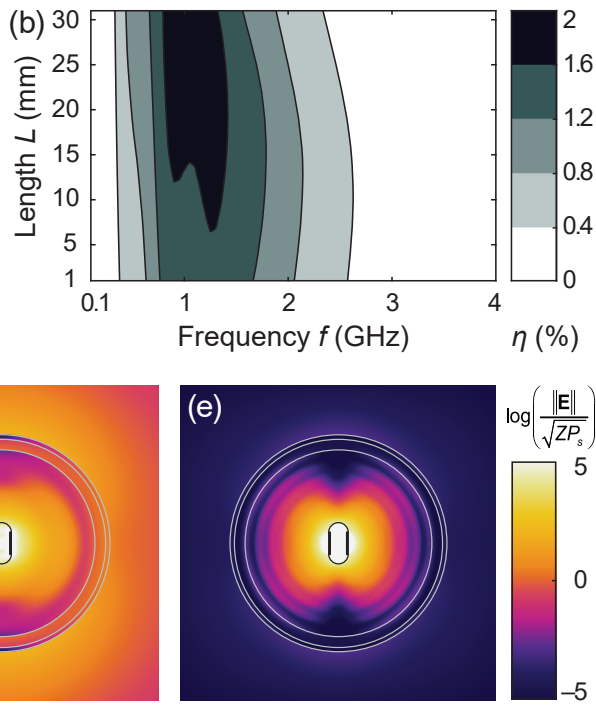

FIG. 3. (a), (b) Theoretical bounds on the radiation efficiency $\eta$ depend on the frequency and the source geometry: (a) $\mathrm{TM}_{10}$ mode and (b) $\mathrm{TE}_{10}$ mode sources centered inside of a spherical $R_{P}=$ $50 \mathrm{~mm}$ phantom $\Omega_{P}$ (dispersive muscleequivalent EM properties, no stratification). (c)-(e) Normalized E-field distributions of the $\mathrm{TM}_{10}$ mode source $(L=$ $10 \mathrm{~mm}, R_{C}=4 \mathrm{~mm}$, and $\left.T=0.5 \mathrm{~mm}\right)$ in a $R_{P}=50 \mathrm{~mm}$ stratified $\Omega_{P}$ at (c) $100 \mathrm{MHz}$ (low $\eta$ due to an inefficient source), (d) $1.2 \mathrm{GHz}$ (maximum $\eta$ ), and (e) $3.5 \mathrm{GHz}$ (low $\eta$ due to attenuation).
TABLE I. Mode-dependent coefficients $c_{i}$ of (3) and adjusted coefficient of determination $\bar{R}^{2}$.

\begin{tabular}{cccccc}
\hline & $c_{1}$ & $c_{2}$ & $c_{3}$ & $c_{4}$ & $\bar{R}^{2}$ \\
\hline $\mathrm{TM}_{10}:$ & 0.00318 & 59.82 & 0.3322 & 0.5318 & 0.9725 \\
$\mathrm{TE}_{10}:$ & 0.00675 & 1.252 & 0.3321 & 0.4006 & 0.9769 \\
\hline
\end{tabular}

mated as $f_{\text {opt }}=16.63 R_{P}^{-0.659}(\mathrm{GHz})$ (adjusted coefficient of determination $\bar{R}^{2}=0.988$ ), and the peak efficiency as $\max (\eta)=0.223 \exp \left(-0.037 R_{P}\right), \bar{R}^{2}=0.984$. For $\mathrm{TE}_{10}, f_{\text {opt }}=17.21 R_{P}^{-0.695}, \bar{R}^{2}=0.979$, and $\max (\eta)=$ $0.191 \exp \left(-0.047 R_{P}\right), \bar{R}^{2}=0.991$.

For a given implantation depth, the $\mathrm{TM}_{10}$ mode allows for higher $\max (\eta)$ compared to $\mathrm{TE}_{10}$. The magnitude of this effect is inversely proportional to $R_{P}: \max \left(\eta_{T M}\right)$ is $78 \%$ higher than $\max \left(\eta_{T E}\right)$ at $R_{P}=20 \mathrm{~mm}$ but approaches the same level at $R_{P}=100 \mathrm{~mm}$ [Fig. 2(c)]. However, $\mathrm{TE}_{10}$ provides substantially better efficiency for $f \lesssim 0.7 \mathrm{GHz}$ (this is consistent with findings of Kim et al. [16]). In addition, there is no substantial effect of $R_{P}$ on $\eta_{T E}$ for $f \lesssim 0.2 \mathrm{GHz}$ [Fig. 2(e)]. Clearly, at such frequencies both sources operate in near field within $\Omega_{P}$. The near field of the $\mathrm{TE}_{10}$ source is essentially magnetic [Fig. 2(b)]; as nearly all biological tissues are weakly diamagnetic, the losses in $\Omega_{P}$ are negligible for this case.

We use $R_{P}=50 \mathrm{~mm}$ as the reference value. In addition to the phantom size, the layers of fat $\left(\varepsilon_{r, 2}, \sigma_{2}\right)$ and skin $\left(\varepsilon_{r, 3}, \sigma_{3}\right)$ [Fig. 1(a), "2" and "3," respectively] affect $\eta$ compared to the homogeneous muscle $\left(\varepsilon_{r, 1}, \sigma_{1}\right)$ phantom of the same $R_{P}$. As $\varepsilon_{r, 1}>\varepsilon_{r, 2} \forall f \in$ $\left[10^{7}, 10^{10}\right] \mathrm{Hz}[23]$, this effect is in part due to mitigation of the wave-impedance contrast with surrounding free space. Obviously, if we add the skin and fat layers inside of $R_{P}=50 \mathrm{~mm}$ sphere, the effect is stronger because of reduced energy dissipation in $\Omega_{P}$ as $\sigma_{1}>\sigma_{2} \forall f$. This case results in the highest $\eta$ for both sources [Fig. 2(f), "inr"]. However, adding the layers outisde of the sp- here [i.e. total $R_{P}=57 \mathrm{~mm}$, Fig. 2(f), "otr"] improves $\eta$ as well compared to the homogeneous case [Fig. 2(f), "hg"]. This happens despite the added attenuation due to $\sigma_{2}$ and $\sigma_{3}$. Since the homogeneous muscle-equivalent phantom gives the most conservative value of $\max (\eta)$, we proceed with the homogeneous $R_{P}=50 \mathrm{~mm}$ case for the subsequent study of different $\Omega_{S}$ configurations. In addition, this setup can be easily replicated for experiments [24].

In terms of $\eta$, spherical sources are optimal for a given $k a$ [14] but impractical for application in bioelectronics. We start by comparing $\eta$ of the cylindrical $\Sigma_{C}$ with the equivalent spherical source: i.e. radius $=a$, same $\varepsilon_{r, S}$ and $T$ [Fig. 1(b)]. $\mathbf{J}_{S}$ is defined according to (1), where $L=\pi a$ for the spherical $\mathrm{TM}_{10}$ source. For spherical sources, $\max (\eta)$ increases $16.9 \%$ for $\mathrm{TM}_{10}$ and $11.0 \%$ for $\mathrm{TE}_{10} ; f_{\text {opt }}$ range remains invariant.

Peak radiation efficiency strongly depends on the dimensions of $\Sigma_{C}$. We compute the effect of both length $L$ and radius $R_{C}$ on $f_{\text {opt }}$ and $\max (\eta)$. Among the parameters of $\Sigma_{C}, L \in[1,31] \mathrm{mm}$ has the strongest effect on $\max (\eta)$. Figs. 3(a) and 3(b) demonstrate this for $\mathrm{TM}_{10}$ and $\mathrm{TE}_{10}$ modes, respectively. Eq. (3) summarizes the observed radiation efficiency $\eta$ results for both modes:

$$
\max [\eta(f, L)]=\frac{c_{1} \ln \left(f+c_{2}\right)}{c_{3} L} \exp \left(-\frac{\ln L-c_{3}}{2 c_{4}^{2}}\right),
$$

where $c_{i}$ are the mode-dependent coefficients (Table I), $L$ is the length $(\mathrm{mm})$, and $f$ is the frequency $(\mathrm{GHz})$.

For $\mathrm{TM}_{10}$, the results are straightforward: the longer the source is, the higher the $\max (\eta)$. For the longest considered one ( $L \approx 3 \mathrm{~cm}$, typical size of a pacemaker or a neural stimulator), $\max (\eta)$ approaches $5 \%$ at $f_{\text {opt }} \approx 1.1 \mathrm{GHz}$ [Fig. 3(a)]. This is about an order of magnitude improvement of $\eta$ compared to existing devices with maximum efficiencies of about $0.5 \%$ [25].

Contrast this with $\mathrm{TE}_{10}$ [Fig. 3(b)]. Here, the efficiency peaks around $1.5 R_{C}<L<3 R_{C}$ that is the opti- 

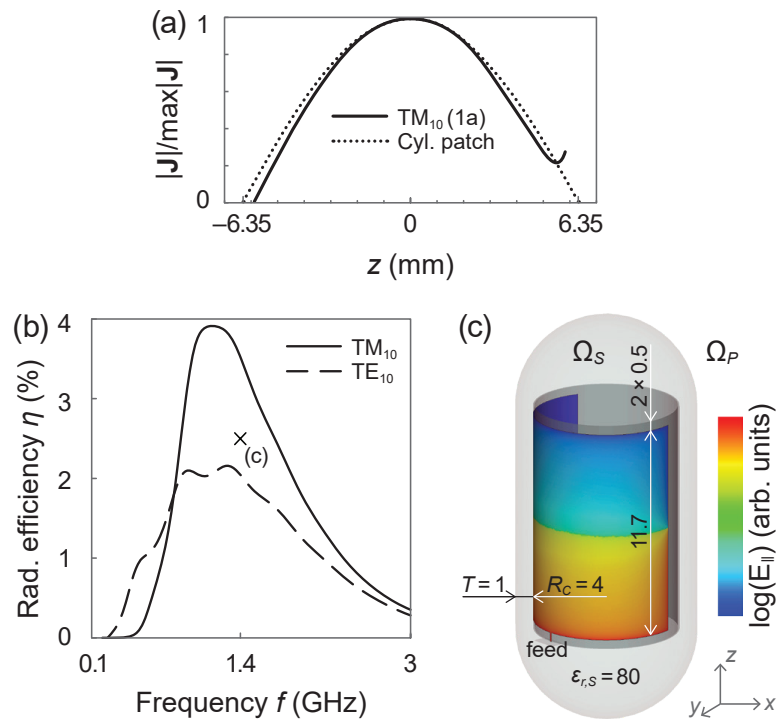

FIG. 4. (a) Cylindrical patch closely replicates surface current density of $\mathrm{TM}_{10}$ source [Eq. (1a)]. Note the rise at $z>5 \mathrm{~mm}$ due to the feed. (b) The dielectric-loaded cylindrical patch efficiency $(x)$ exceeds the theoretical bounds of $\mathrm{TE}_{10}$ and closely approaches $\eta$ of $\mathrm{TM}_{10}$. (c) Synthesized cylindrical patch design $(\mathrm{mm})$ and time snapshot of E-field distribution.

mal dimension range for inductor sources. We shall also see that for $L \lesssim 5 \mathrm{~mm}$ the $\mathrm{TE}_{10}$ mode not only gives better $\max (\eta)$ but also improved $\eta$ for $f<f_{\text {opt }}$. At these frequencies, an electrically-small $\mathrm{TM}_{10}$ source (e.g. $L=10 \mathrm{~mm} \approx \lambda / 37$ at $100 \mathrm{MHz}$ in muscle) produces a weak E-field [Fig. 3(c)]. Consequently, $\mathrm{TM}_{10}$ is inefficient under these conditions. On the other hand, the same $\mathrm{TM}_{10}$ source operating at $f_{\text {opt }} \approx 1.2 \mathrm{GHz}$ [Fig. 3(d)] results in $55 \%$ increase in $\max (\eta)$ compared to the $\mathrm{TE}_{10}$ one. In this case, $L \approx \lambda / 3$.4. For $f>f_{\text {opt }}$, the decline of $\eta$ is nearly identical for both modes as it is driven by the attenuation of the wave propagating inside $\Omega_{P}$ [Fig. 3(e)].

The radius of $\Sigma_{C}$ [Fig. 1(b)] has a moderate effect on radiation. For $R_{C} \in[1,5] \mathrm{mm}$, the optimal frequency remains invariant for both modes: $f_{\text {opt }}=1.3 \mathrm{GHz}$ $\pm 50 \mathrm{MHz}$. However, $\max (\eta)$ is linearly proportional to $R_{C}(\mathrm{~mm})$. Given $L=10 \mathrm{~mm}, \mathrm{TM}_{10}$ yields $\max (\eta)=$ $3.7 \times 10^{-3} R_{C}+0.017\left(\bar{R}^{2}=0.9902\right)$, and $\mathrm{TE}_{10}$ gives $\max (\eta)=1.9 \times 10^{-3} R_{C}+0.013\left(\bar{R}^{2}=0.9956\right)$. For the studied radii, $\max (\eta)$ is roughly twice for $\mathrm{TM}_{10}$. But, again, $\mathrm{TE}_{10}$ results in better $\eta$ in the sub-GHz range.

Next, we characterize the effect of $\Omega_{S}$ dielectric loading on $\eta$ using $\varepsilon_{r, S}(\omega)=c_{d l} \varepsilon_{r, 1}(\omega)$ [Fig. 1(b)], where $\varepsilon_{r, 1}$ is the dispersive permittivity of muscle and $c_{d l} \in$ $\left[1 / \varepsilon_{r, 1}, 2\right]$ is the coefficient. $c_{d l}=1 / \varepsilon_{r, 1} \Rightarrow \Omega_{S}\left\{\varepsilon_{0}, 0\right\}$ that represents the source without any artificial dielectric loading (i.e. only surrounding tissues load the source; the dependence of tissue EM properties on $\eta$ was studied in [19]). Given the definition of sources through $\mathbf{J}_{s}$ [see Eq. (1)], the dielectric loading impacts only the efficiency of the $\mathrm{TM}_{10}$ source and has no effect on the $\mathrm{TE}_{10}$ one. For $\mathrm{TM}_{10}, \varepsilon_{r, S}$ is inversely proportional to the optimal frequency $(\mathrm{GHz})$ as $f_{\text {opt }}=$ $-5.63 \times 10^{-2} c_{d l}+1.34\left(\bar{R}^{2}=0.884\right)$. Dielectric loading of $\mathrm{TM}_{10}$ source significantly improves radiation performance: $\max (\eta)=4.3 \times 10^{-3} c_{d l}+0.027\left(\bar{R}^{2}=0.943\right)$. Note that the latter term is $\max (\eta)$ of an unloaded $\mathrm{TM}_{10}$ source. These effects are in part due to the scaling of the source electrical size $k a \propto \sqrt{\varepsilon^{\mathrm{eff}}}$, where $\varepsilon^{\mathrm{eff}}$ is the effective permittivity around the source, which is function of $\varepsilon_{r, S}$.

A high-permittivity shell of thickness $T \in[0.1,3] \mathrm{mm}$ can be used to artificially load the source (i.e. via $\varepsilon_{r}, S$ ) to increase $\eta$ (biocompatibility of the shell must be ensured through in vitro and in vivo tests in accordance with current regulations). In addition, the low-loss shell reduces dissipation in the near-field of the $\mathrm{TM}_{10}$ source. Given $\varepsilon_{r, S} \approx \varepsilon_{r, 1}$ (wave impedance is closely matched with a surrounding tissue), $T$ affects the optimal frequency insignificantly ( $f_{\text {opt }} \approx 1.2 \pm 0.1 \mathrm{GHz}$ for both sources). However, $\eta \propto T$, and the effect on $\mathrm{TM}_{10}$ mode is stronger: $\max (\eta)=6.1 \times 10^{-3} T+0.029\left(\bar{R}^{2}=1\right)$. For $\mathrm{TE}_{10}$, $\max (\eta)=1.8 \times 10^{-3} T+0.02\left(\bar{R}^{2}=1\right)$. These results are consistent with the findings of Merli et al. [26]. Note that if $\varepsilon_{r, S} \neq \varepsilon_{r, 1}$, thicker shell would also affect the optimal frequency by increasing the effect of $\varepsilon_{r, S}$ described above. So, $f_{\text {opt }} \propto 1 /\left(\varepsilon_{r, S} \cdot T\right)$.

Optimal Source Realization-Taking into account on the identified features, we design a proof-of-concept body-implanted capsule (Fig. 4) that shows about fivefold improvement of radiation efficiency compared to existing devices [25]. Having $\eta \approx 2.5 \%$, it exceeds the theoretical bounds of $\mathrm{TE}_{10}$ and closely approaches $\eta$ of the $\mathrm{TM}_{10}$ source [Fig. 4(b)]. The capsule operates at Wireless Medical Telemetry Service (WMTS) band centered around $f_{0}=1.4 \mathrm{GHz}$ that is close to $f_{\text {opt }}$ [Fig. 4(b)].

Fig. 4(c) shows the synthesized cylindrical-patch structure that is the most suitable to closely replicate the theoretical $\mathrm{TM}_{10}$ mode source [Fig. 2(a)] in terms of $\mathbf{E}$ and $\mathbf{J}_{s}$ distributions [Fig. 4(a)]. The capsule shell $(T=1 \mathrm{~mm})$ and 76.2 - $\mu \mathrm{m}$-thick $(3 \mathrm{mil})$ substrate load the antenna with $\varepsilon_{r, S}=80$ that is close to maximum permittivity of human tissues as well as water at $1.4 \mathrm{GHz}$ [23]. The source radius is $R_{C}=4 \mathrm{~mm}$. Within this environment and considering $\Omega_{P}$, the $\lambda / 2$ resonant length (i.e. when $\left.\Im\left[Z_{A}\left(f_{0}\right)\right]=0\right)$ of the patch is $11.7 \mathrm{~mm}$. The ground plane extends $0.5 \mathrm{~mm}$ further to mitigate $\mathbf{E}$-field fringing to the inside of the capsule. Therefore, the total $\Sigma_{C}$-equivalent length is $L=12.7 \mathrm{~mm}$, which was used to calculate the theoretical bounds in Fig. 2(a).

The cylindrical patch spans $\Sigma_{C}$ over a sector angle $\varphi_{C}$ in the azimuth plane $x-y$. As the physical size increases with the angle, $\eta \propto \varphi_{C} \in(0, \pi]$. However, an array of narrow sources (i.e. $\varphi_{C} \ll \pi$ ) makes it possible to realize superdirective beamforming [27]. Along with the adaptive frequency hopping to adjust for varying implantation depth [17], such source minimizes the energy dissipation 
in tissues therefore maximizing $\eta$.

Conclusion - Optimal radiation performance requires minimizing the dissipated energy for a given power flow in the far field. For a given radiation pattern, the maximum operating range and data rates of body-implanted capsules can only be improved by increasing the radiation efficiency. In this Letter, we quantified the tradeoffs between the parameters of $\mathrm{TM}_{10}$ and $\mathrm{TE}_{10}$ sources and their achievable efficiencies so that optimal radiation can be achieved. The established physical bounds on $\eta$ serve as the design quality gauge, facilitate the choice of the source type and dimensions, and provide simple rules to check the feasibility of a given design. While the study uses simple tissue structures, Ref. [17] suggests that the findings remain valid in realistic tissue geometries.

Based on the established features, we showed that the fundamental bounds exceed by far the efficiencies obtained by conventional designs. We closely approach these bounds in practice by using a dielectric-loaded radiating structure that closely replicates $\mathbf{J}_{s}$ distribution of the $\mathrm{TM}_{10}$ source. The optimized source outperforms the existing systems by a factor of five, which represents a fundamentally new capability enabling safe, powerefficient, and high throughput devices.

This work was supported in part by the European Union through the ERD Fund and by the French region of Brittany, Ministry of Higher Education and Research, Rennes Métropole and Conseil Départemental 35, through the CPER Project SOPHIE / STIC \& Ondes.

* d@deniq.com

[1] A. S. Y. Poon, "Miniaturized biomedical implantable devices," in Implantable Bioelectronics (Wiley-VCH, Weinheim, Germany, 2014) pp. 45-64.

[2] K. Famm, B. Litt, K. Tracey, E. Boyden, and M. Slaoui, "Drug discovery: A jump-start for electroceuticals," Nature 496, 159-161 (2013).

[3] Y. Urzhumov and D. R. Smith, "Metamaterial-enhanced coupling between magnetic dipoles for efficient wireless power transfer," Phys. Rev. B 83, 205114 (2011).

[4] S. Kim, J. S. Ho, L. Y. Chen, and Ada S. Y. Poon, "Wireless power transfer to a cardiac implant," Appl. Phys. Lett. 101, 073701 (2012).

[5] A. S. Rekhi and A. Arbabian, "Remote sub-wavelength focusing of ultrasonically activated Lorentz current," Appl. Phys. Lett. 110, 164104 (2017).

[6] D. Agrawal et al., "Conformal phased surfaces for wireless powering of bioelectronic microdevices," Nat. Biomed. Eng. 1, 0043 (2017).

[7] J. Jun et al., "Fully integrated silicon probes for highdensity recording of neural activity," Nature 551, 232236 (2017).

[8] J. S. Ho, Y. Tanabe, S. M. Iyer, A. J. Christensen, L. Grosenick, K. Deisseroth, S. L. Delp, and A. S. Y. Poon, "Self-tracking energy transfer for neural stimulation in untethered mice," Phys. Rev. Applied 4, 024001 (2015).

[9] H. Takehara, A. Nagaoka, J. Noguchi, T. Akagi, H. Kasai, and T. Ichiki, "Lab-on-a-brain: Implantable microoptical fluidic devices for neural cell analysis in vivo," Sci. Rep. 4, 6721 (2014).

[10] D. Kim, M. Kim, J. Yoo, H.-H. Park, and S. Ahn, "Magnetic resonant wireless power transfer for propulsion of implantable micro-robot," J. Appl. Phys. 117, 17E712 (2015).

[11] L. J. Chu, "Physical limitations of omni-directional antennas," J. Appl. Phys. 19, 1163-1175 (1948).

[12] M. Gustafsson, C. Sohl, and G. Kristensson, "Physical limitations on antennas of arbitrary shape," Proc. Royal Soc. A 463, 2589-2607 (2007).

[13] A. Karlsson, "On the efficiency and gain of antennas," Prog. Electromagn. Res. 136, 479-494 (2013).

[14] C. Pfeiffer, "Fundamental efficiency limits for small metallic antennas," IEEE Trans. Antennas Propag. 65, 1642-1650 (2017).

[15] M. Shahpari and D. V. Thiel, "Fundamental limitations for antenna radiation efficiency," IEEE Trans. Antennas Propag. 66, 3894-3901 (2018).

[16] S. Kim, J. S. Ho, and A. S. Y. Poon, "Midfield wireless powering of subwavelength autonomous devices," Phys. Rev. Lett. 110, 203905 (2013).

[17] D. Nikolayev, M. Zhadobov, P. Karban, and R. Sauleau, "Electromagnetic radiation efficiency of body-implanted devices," Phys. Rev. Applied 9, 024033 (2018).

[18] A. Puzenko, P. B. Ishai, and Y. Feldman, "Cole-Cole broadening in dielectric relaxation and strange kinetics," Phys. Rev. Lett. 105, 037601 (2010).

[19] D. Nikolayev, M. Zhadobov, and R. Sauleau, "Impact of tissue electromagnetic properties on radiation performance of in-body antennas," IEEE Antenn. Wireless Propag. Lett. 17, 1-5 (2018).

[20] J. D. Jackson, Classical electrodynamics, 3rd ed. (John Wiley \& Sons, Hoboken, NJ, 1999).

[21] P. Karban, F. Mach, P. Kus, D. Panek, and I. Dolezel, "Numerical solution of coupled problems using code Agros2D," Computing 95, 381-408 (2013).

[22] S. Gabriel, R. W. Lau, and C. Gabriel, "The dielectric properties of biological tissues: III. Parametric models for the dielectric spectrum of tissues," Phys. Med. Biol. 41, 2271-2293 (1996).

[23] S. Gabriel, R. W. Lau, and C. Gabriel, "The dielectric properties of biological tissues: II. Measurements in the frequency range $10 \mathrm{~Hz}$ to $20 \mathrm{GHz}$," Phys. Med. Biol. 41, 2251-2269 (1996).

[24] D. Nikolayev, M. Zhadobov, L. Le Coq, P. Karban, and R. Sauleau, "Robust ultra-miniature capsule antenna for ingestible and implantable applications," IEEE Trans. Antennas Propag. 65, 6107-6119 (2017).

[25] D. Nikolayev, M. Zhadobov, P. Karban, and R. Sauleau, "Conformal antennas for miniature in-body devices: The quest to improve radiation performance," Radio Science Bulletin 363, 52-64 (2017).

[26] F. Merli, B. Fuchs, J. R. Mosig, and A. K. Skrivervik, "The effect of insulating layers on the performance of implanted antennas," IEEE Trans. Antennas Propag. 59, 21-31 (2011).

[27] S. Arslanagic and R. W. Ziolkowski, "Highly subwavelength, superdirective cylindrical nanoantenna," Phys. Rev. Lett. 120, 237401 (2018). 